\title{
JAK/STAT pathway inhibition overcomes IL7-induced glucocorticoid resistance in a subset of human T-cell acute lymphoblastic leukemias
}

\author{
C Delgado-Martin ${ }^{1,8}$, LK Meyer ${ }^{1,8}$, BJ Huang ${ }^{1}$, KA Shimano $^{1}$, MS Zinter ${ }^{1}$, JV Nguyen $^{1}$, GA Smith ${ }^{2}$, J Taunton $^{2}$, SS Winter $^{3}$, JR Roderick $^{4}$, \\ MA Kelliher ${ }^{4}$, TM Horton ${ }^{5}$, BL Wood ${ }^{6}$, DT Teachey ${ }^{7}$ and ML Hermiston ${ }^{1}$
}

While outcomes for children with T-cell acute lymphoblastic leukemia (T-ALL) have improved dramatically, survival rates for patients with relapsed/refractory disease remain dismal. Prior studies indicate that glucocorticoid (GC) resistance is more common than resistance to other chemotherapies at relapse. In addition, failure to clear peripheral blasts during a prednisone prophase correlates with an elevated risk of relapse in newly diagnosed patients. Here we show that intrinsic GC resistance is present at diagnosis in early thymic precursor (ETP) T-ALLs as well as in a subset of non-ETP T-ALLs. GC-resistant non-ETP T-ALLs are characterized by strong induction of JAK/STAT signaling in response to interleukin-7 (IL7) stimulation. Removing IL7 or inhibiting JAK/STAT signaling sensitizes these T-ALLs, and a subset of ETP T-ALLs, to GCs. The combination of the GC dexamethasone and the JAK1/2 inhibitor ruxolitinib altered the balance between pro- and anti-apoptotic factors in samples with IL7-dependent GC resistance, but not in samples with IL7-independent GC resistance. Together, these data suggest that the addition of ruxolitinib or other inhibitors of IL7 receptor/JAK/STAT signaling may enhance the efficacy of GCs in a biologically defined subset of T-ALL.

Leukemia (2017) 31, 2568-2576; doi:10.1038/leu.2017.136

\section{INTRODUCTION}

T-cell acute lymphoblastic leukemia (T-ALL) arises from malignant transformation of T-cell progenitors in the thymus. ${ }^{1}$ Intensified therapy has dramatically improved survival for pediatric patients. ${ }^{2}$ However, outcomes for patients with relapsed or refractory T-ALL remain dismal, with 5 -year survival rates $<10 \%$. $^{3}$ Unfortunately, biomarkers of high-risk disease that might facilitate rational therapeutic approaches that could be introduced early in therapy are limited. ${ }^{4}$ Importantly, newly diagnosed patients that have positive minimal residual disease (MRD) after initial therapy or that fail to rapidly clear peripheral leukemic blasts during a prednisone prophase have poorer outcomes. ${ }^{5,6}$ These data suggest that intrinsic variability in sensitivity to chemotherapy and specifically to glucocorticoids (GCs) exists at diagnosis. The observation that GC resistance is commonly present at relapse ${ }^{7}$ and is more frequent than is resistance to other drugs $^{8}$ further supports this idea and suggests that enhancing GC sensitivity in high-risk patients early in therapy could have therapeutic benefit.

GCs bind to the cytoplasmic GC receptor (GR) to form a complex that translocates to the nucleus, where it regulates genes implicated in diverse cellular processes including cell cycle arrest and apoptosis. 9,10 Insights into the mechanistic basis of GC resistance in T-ALL are limited, and this is a barrier to implementing rational therapeutic strategies for preventing or overcoming it. Whereas alterations in GR function are a frequent cause of GC resistance in T-ALL cell lines, similar abnormalities are rare in patients. ${ }^{11,12}$ Leukemogenic events such as AKT hyperactivation ${ }^{13}$ and $I k z f 1$ mutations $^{14}$ have been implicated in GC resistance in a subset of patients. Addition of interleukin-7 (IL7) has also been shown to induce GC resistance in vitro in a subset of samples. ${ }^{15}$ However, it is uncertain how these findings might be translated into actionable therapeutic interventions.

The genetic heterogeneity of T-ALL has precluded the use of genetic alterations for risk-based stratification. We reasoned that these diverse genetic lesions might converge on a more limited set of biochemical abnormalities that could be used to identify subsets of T-ALLs that share common mechanisms of chemotherapy resistance. We tested this hypothesis by assessing drug responses in vitro using phosphoflow cytometry in primary T-ALL cells. Here, we show that intrinsic GC resistance is a hallmark of T-ALLs arising at the early thymic precursor (ETP) stage and also characterizes a subset of non-ETP T-ALLs. GC-resistant non-ETP T-ALLs can be identified by augmented JAK/STAT signaling in response to IL7 stimulation. Removing IL7 from the media sensitizes these samples, and a subset of ETP T-ALLs, to GCs, but not to other chemotherapies. Interestingly, only 4 of the 32 samples (12.5\%) used in this study had mutations in the IL7 receptor (IL7R)/JAK/STAT pathway, suggesting that IL7-induced GC resistance in this subset of $T-A L L$ is independent of genetic drivers of pathway activity and instead reflects a shared biologic property that can be functionally defined. The addition of the clinically available JAK1/2 inhibitor ruxolitinib or newly developed JAK3

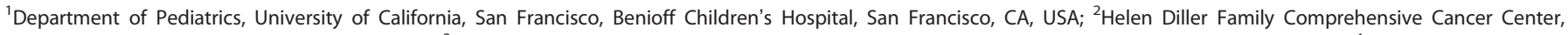

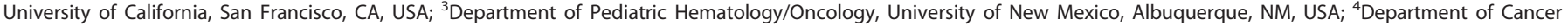

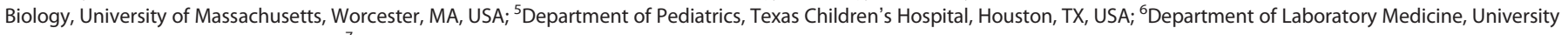

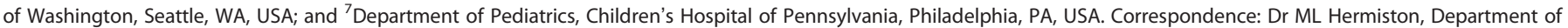
Pediatrics, University of California, San Francisco, Benioff Children's Hospital, 1450 3rd Street Room HD250, San Francisco 94158, CA, USA.

E-mail: michelle.hermiston@ucsf.edu

${ }^{8}$ These authors contributed equally to this work.

Received 28 October 2016; revised 9 March 2017; accepted 24 April 2017; accepted article preview online 9 May 2017; advance online publication, 30 May 2017
} 
inhibitor reversed intrinsic GC resistance. Together, these studies support the use of JAK inhibitors to increase the efficacy of GCs in a biologically defined subset of T-ALL patients.

\section{MATERIALS AND METHODS}

\section{Patient samples and patient derived xenografts}

Diagnostic bone marrow samples were obtained from sources indicated in Supplementary Table 1. Informed consent for use of diagnostic specimens for future research was obtained from patients or their guardians at the time of sample collection, according to the Declaration of Helsinki, the National Cancer Institute, and institutional review boards of participating sites. The Institutional Animal Care and Use Committee approved animal studies. Characteristics of patient derived xenografts (PDXs) are listed in Supplementary Table 1. ETP status was defined in the COG central reference laboratory as previously described. ${ }^{4}$ PDXs were established by injecting 1.5 to $2.5 \times 10^{6}$ cells intravenously into non-obese diabetic/severe combined immunodeficient NOD/SCID/II $2 \mathrm{rg}^{t \mathrm{~m} / \mathrm{wj} /} / \mathrm{SzJ}$ (NSG) mice as previously described. ${ }^{16}$ Engraftment was determined by flow cytometric analysis of peripheral blood using anti-human CD45 antibodies. PDX samples were used between passages 1 and 3 and evaluated at each passage for fidelity of cell surface markers, biochemical signatures, and drug responsiveness.

\section{Drug treatment and phosphoflow cytometry}

Cells were thawed and rested $1 \mathrm{~h}$ at $37{ }^{\circ} \mathrm{C}$ in complete media (RPMI, $10 \%$ FBS, $1 \%$ penicillin/streptomycin, $1 \%$ Glutamax (ThermoFisher, Waltham, MA, USA)). $10^{6}$ cells $/ \mathrm{ml}$ were cultured in a 96-well plate with vehicle (DMSO) or drug (araC, dexamethasone, etoposide, methotrexate, vorinostat (Sigma-Aldrich, St Louis, MO, USA), vincristine (Hospira, Lake Forest, IL, USA), ruxolitinib and MK2206 (Selleck, Houston, TX, USA) and/or a newly developed JAK3 inhibitor $\left.(\text { JAK3i })^{17}\right)$. Since spontaneous cell death is common with in vitro culture of T-ALL samples, media was supplemented with $25 \mathrm{ng} / \mathrm{ml}$ IL7 (Peprotech, Rocky Hill, NJ, USA), a cytokine known to inhibit spontaneous apoptosis in T-ALL, ${ }^{18}$ unless otherwise indicated. Cells were collected at $48 \mathrm{~h}$ and flow cytometry performed as previously described ${ }^{19}$ using a FacsVerse flow cytometer (BD Biosciences, San Jose, CA, USA). Antisera included anti-human CD7, activated caspase-3, phospho-STAT5 (pSTAT5), phospho-Akt (pAkt) (Cat\# 564019, 560627, 612599 and 560404, BD Biosciences), CD45, IL7R (Cat\# 25-0459 and 201278, Tonbo Biosciences, San Diego, CA, USA), BIM, GR (Cat\# 2933 and 12041, Cell Signaling, Danver, MA, USA) and BCL2 (Cat\# MHBCL04, ThermoFisher). The frequency of viable Hoechst (ThermoFisher, Ashland, OR, USA) or active caspase-3 negative cells in the CD7-positive gate was determined using FlowJoV9.8.2 (TreeStar, Ashland, OR, USA). For phosphoflow studies, only viable CD7-positive cells were analyzed.
BH3 profiling

Apoptotic priming was measured as the depletion of intracellular cytochrome $\mathrm{C}$ using flow cytometry-based $\mathrm{BH} 3$ profiling as described by Ryan et al. ${ }^{19}$ Briefly, $10^{6}$ cells $/ \mathrm{ml}$ in complete media containing $25 \mathrm{ng} / \mathrm{ml}$ IL7 were treated with vehicle, $1 \mu \mathrm{m}$ dexamethasone and/or $500 \mathrm{~nm}$ ruxolitinib for $16 \mathrm{~h}$, permeabilized with digitonin (Sigma-Aldrich), exposed to $100 \mathrm{nM}$ BIM BH3 peptide (New England Biosciences, Ipswich, MA, USA) or 1 M M ABT-199 (ApexBio, Houston, TX, USA) for $1 \mathrm{~h}$, fixed with $4 \%$ paraformaldehyde, stained with anti-cytochrome $c$ antibody (Cat\# 558709, BD Biosciences), and subjected to flow cytometry. Cells treated with DMSO and alamethicin (Alfa Aesar, Haverhill, MA, USA) were used as negative and positive controls respectively. For each condition, the percentage of cytochrome $c$ depletion was calculated based on the release of cytochrome $c$ in the DMSO ( $0 \%$ loss) and alamethicin ( $100 \%$ loss) controls and then normalized to vehicle treated cells.

\section{Statistical analysis}

Statistical analyses, as defined in each figure legend, were performed using Prism V5.0 (GraphPad, La Jolla, CA, USA).

Additional information is available in Supplementary Methods.

\section{RESULTS}

Intrinsic GC sensitivity identifies distinct T-ALL subsets

Previous studies demonstrated that GC resistance in diagnostic T-ALL samples in vitro correlated with poor response to upfront GC treatment. $^{20-22}$ To ask whether this in vitro resistance is also associated with failure to eradicate circulating blasts by the end of the induction phase of therapy, we exposed diagnostic samples obtained from patients treated on the COG protocol AALL0434 to vehicle control or dexamethasone and measured the percentage of viable cells $48 \mathrm{~h}$ later. Whereas leukemic blasts from patients with detectable MRD (MRD+) were uniformly resistant to GCs, cells from most MRD- patients were GC sensitive (Supplementary Figure S1).

Many PDX models accurately recapitulate the clinical features of the cancers from which they were derived. ${ }^{23,24}$ To obtain sufficient numbers of primary blasts for detailed mechanistic studies, we employed this model system to expand 22 non-ETP and 10 ETP T-ALL samples derived from newly diagnosed pediatric T-ALL patients in NSG mice (Supplementary Table 1). In vitro exposure to dexamethasone reduced the viability of all ETP T-ALL by $<50 \%$. In contrast, the 22 non-ETP T-ALLs exhibited significant variability in GC sensitivity, with 5 being highly GC sensitive and 8 others
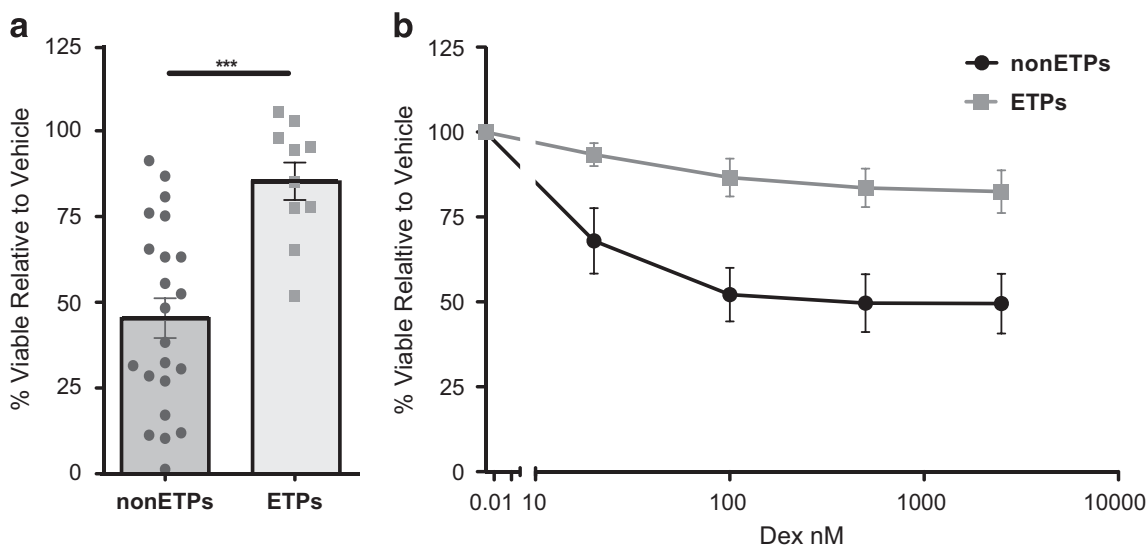

Figure 1. ETP T-ALL samples are intrinsically more resistant to GCs than are non-ETP T-ALLs. PDX samples were thawed, rested $1 \mathrm{~h}$, and then exposed to vehicle or dexamethasone (Dex). Cells were stained with Hoechst dye at $48 \mathrm{~h}$ and subjected to flow cytometry. (a) Percentage of viable cells, relative to vehicle, of non-ETP T-ALL (circles, $n=22$ ) and ETP T-ALL (squares, $n=10$ ) PDX samples after $48 \mathrm{~h}$ treatment with $2.5 \mu \mathrm{m}$ dexamethasone. Data were analyzed using the parametric unpaired $t$-test with Welch's correction (unequal variances). ${ }^{* * *} P<0.001$. (b) Percentage of viable cells, relative to vehicle, of representative samples of ETP T-ALLs $(n=8)$ and non-ETP T-ALLs $(n=10)$ exposed to increasing doses of dexamethasone $(0,100,500 \mathrm{~nm}$ and $2.5 \mu \mathrm{m})$ for $48 \mathrm{~h}$. Data are represented as mean \pm s.e.m. of the biological replicates. Error bars show s.e.m. 
showing ETP-like resistance to dexamethasone-induced apoptosis (Figure 1a). Higher concentrations of dexamethasone failed to further affect viability, suggesting that individual leukemias have

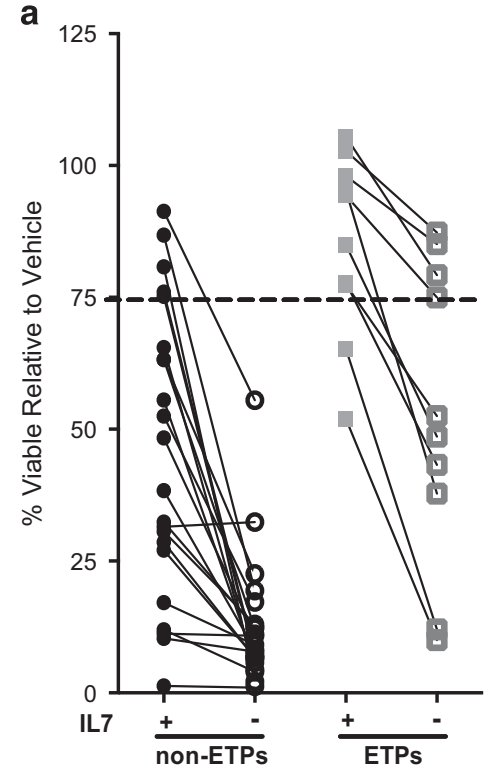

b

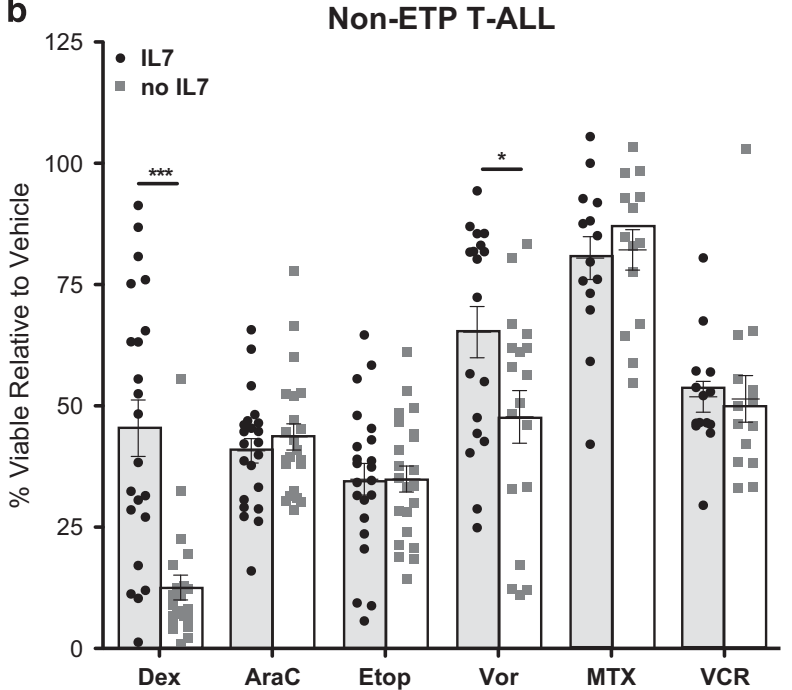

C

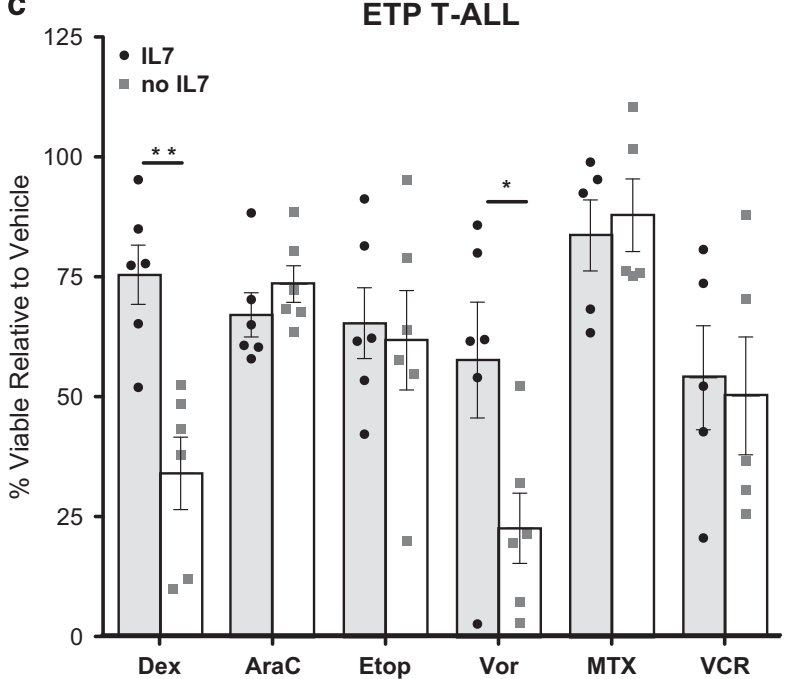

intrinsic set points for GC sensitivity (Figure 1b). These differences in GC sensitivity are not due to variable levels of GR expression (Supplementary Figure S2).

IL7 mediates GC resistance in a subset of T-ALL

We previously demonstrated that ETP T-ALLs and a subset of nonETP T-ALLs activate downstream signaling molecules in response to IL7 stimulation regardless of the presence of an IL7R pathway mutation. ${ }^{16}$ Furthermore, the JAK $1 / 2$ inhibitor ruxolitinib inhibits this response and prevents leukemic outgrowth of ETP T-ALLs in a PDX model. ${ }^{16}$ To determine whether IL7 modulates intrinsic GC sensitivity in T-ALL blasts, we treated PDX samples with vehicle or dexamethasone in the presence or absence of IL7. As expected given the pro-survival function of IL7, ${ }^{25,26} \mathrm{~T}$-ALL cell viability was significantly reduced in many samples cultured in the absence of IL7 (Supplementary Figure S3). A subset of ETPs remained GC resistant in the absence of IL7, suggesting IL7-independent mechanisms of GC resistance in these leukemias. Intriguingly, however, the remaining leukemias exhibited greatly enhanced GC sensitivity upon removal of IL7 (referred to henceforth as 'IL7dependent GC-resistant') (Figure 2a). Consistent with our prior finding, ${ }^{16}$ sequencing of IL7R, JAK1, JAK3, STAT5A, STAT5B and $D N M 2$ revealed that IL7-dependent GC resistance is independent of the presence of an activating IL7R/JAK/STAT pathway mutation (Supplementary Table 1).

The protective effect of IL7 is drug-specific

Since chemotherapy regimens for ALL consist of multiple drugs, ${ }^{27}$ we investigated if the ability of IL7 to protect T-ALL cells from chemotherapy-induced death was GC specific or reflective of panchemotherapy resistance. To better understand the mechanistic basis for this protective effect of IL7, we exposed non-ETP and IL7dependent GC-resistant ETP T-ALL PDX samples to conventional chemotherapeutic agents in the presence of IL7. IL7 failed to protect them against death induced by cytosine arabinoside, methotrexate, vincristine, or etoposide (Figures $2 \mathrm{~b}$ and $\mathrm{c}$ ). As expected, IL7 protected both non-ETP and ETP T-ALLs from death induced by dexamethasone. Interestingly, IL7 treatment also antagonized the ability of vorinostat, a histone deacetylase (HDAC) inhibitor, to kill T-ALL cells in vitro (Figures $2 \mathrm{~b}$ and $\mathrm{c}$ ).

IL7 responsiveness distinguishes a subset of GC-resistant non-ETP T-ALLS

To determine if IL7 responsiveness might serve as a biomarker to distinguish GC sensitive and GC-resistant non-ETP T-ALLs, we stimulated non-ETP T-ALL PDX samples with IL7 and measured changes in phosphorylated STAT5 (pSTAT5) levels (Supplementary Figure S4A). These studies indicate that non-ETP T-ALLs can be broadly assigned to one of three groups based on their IL7 response: (1) a highly responsive subset with pSTAT5 induction

Figure 2. IL7 deprivation sensitizes a subset of T-ALL samples to dexamethasone-induced cell death. (a) Percentage of viable cells, relative to vehicle, after $48 \mathrm{~h}$ of treatment with $2.5 \mu \mathrm{m}$ dexamethasone in the presence (filled shapes) or absence (empty shapes) of $25 \mathrm{ng} / \mathrm{ml} \mathrm{IL7}$. Dashed line indicates 75\% relative viability. Non-ETP T-ALL $(n=22)$, ETP T-ALL $(n=10)$. (b) Non-ETP and (c) ETP PDX samples were subjected to $48 \mathrm{~h}$ treatment with $2.5 \mu \mathrm{m}$ dexamethasone, $125 \mathrm{ng} / \mathrm{ml} \mathrm{AraC,} 500 \mathrm{~nm}$ etoposide (etop), $250 \mathrm{~nm}$ vorinostat (vor), $2.5 \mu \mathrm{m}$ methotrexate (MTX), or $5 \mu \mathrm{m}$ vincristine (VCR) in the presence (gray) or absence (white) of $25 \mathrm{ng} / \mathrm{ml} \mathrm{IL7}$ and processed as above ( $n=4$ to 22 ). Paired T-test (Wilcoxon-non normal distribution-in the case of ETPs) was used to evaluate statistical significance. ${ }^{*} P<0.05 ;{ }^{* *} P<0.01 ;{ }^{* * *} P<0.001$. Data are represented as mean \pm s.e.m. of the biological replicates. Error bars show s.e.m. 
similar to ETP T-ALL samples (responders; $n=10$ ); (2) a subset with a smaller biphasic shift in pSTAT5 (partial responders; $n=7$ ); and (3) a subset with no response (non-responders; $n=5$ ) (Figure 3a). We next asked if IL7 responsiveness predicts GC sensitivity in nonETP T-ALLs by plotting the GC sensitivity of these three subsets according to the ability of IL7 to increase PSTAT5 levels (Figure $3 \mathrm{~b}$ ). The non-ETP responder subset displayed similar intrinsic resistance to GC-induced death as did IL7-dependent GCresistant ETP T-ALLs, while the non-responder subset was most GC sensitive. Partial responders exhibited an intermediate phenotype. Removing IL7 from the media markedly enhanced cell death in the responder and partial responder groups but had no impact on the non-responder group (Figure $3 \mathrm{~b}$ ). Moreover, stratifying nonETP T-ALL samples according to their IL7 response and evaluating their sensitivity to the drugs tested in Figure 2 showed that

\section{a}

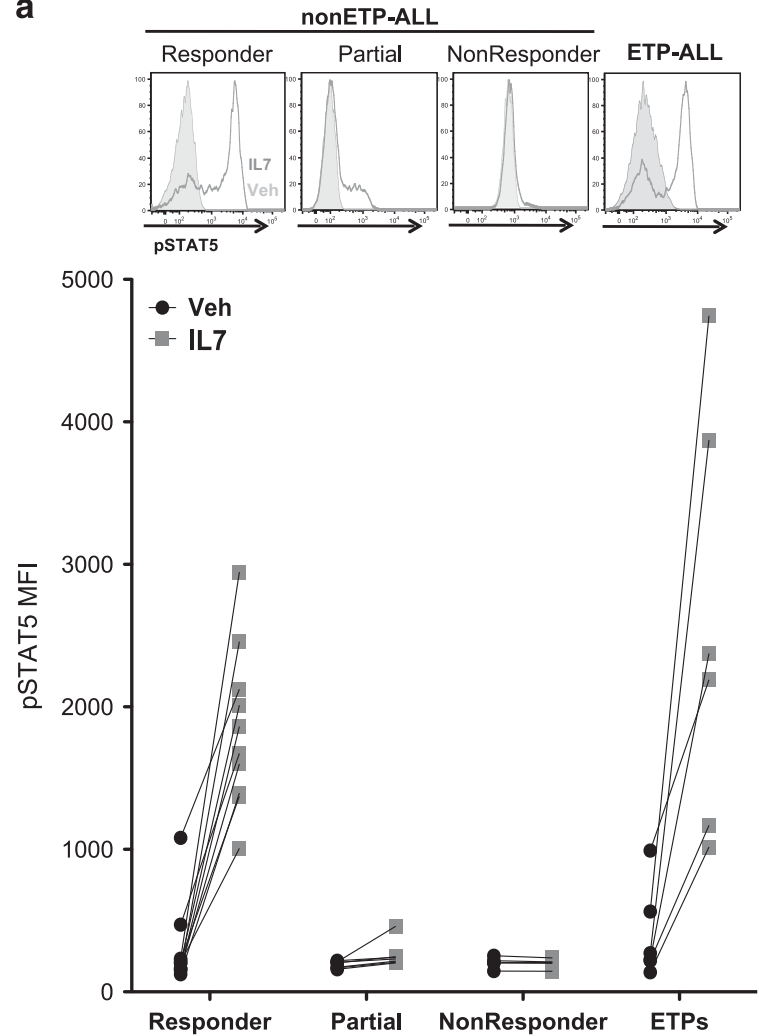

b

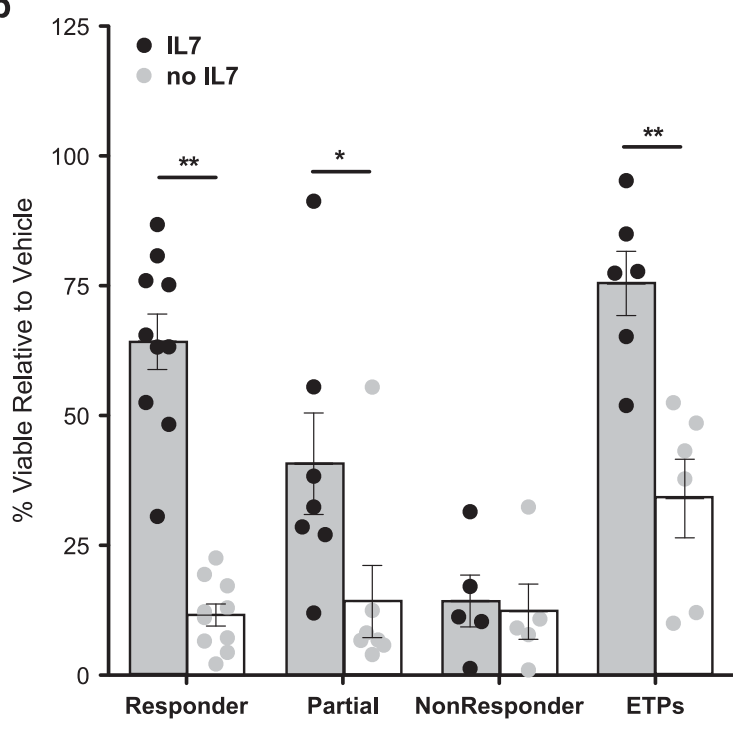

responders behaved similarly to ETP T-ALLs, with IL7-dependent resistance to dexamethasone and vorinostat, but that IL7 had no effect on drug sensitivity for non-responders (Supplementary Figure S4B).

We previously reported that IL7 responsiveness correlated with IL7R expression in ETP and non-ETP T-ALLs. ${ }^{16}$ Similarly, plotting the difference in PSTAT5 levels between basal and IL7-stimulated cells ( $\triangle$ pSTAT5) against IL7R expression in this extended panel of T-ALLs confirmed a significant positive relationship between these parameters (Supplementary Figure S4C, right). There was also a positive correlation between $\triangle$ PSTAT5 and sensitivity to GCs in the presence of IL7 (Supplementary Figure S4C, left). Together, these data indicate that intrinsic GC sensitivity at diagnosis is modulated by IL7 in a subset of T-ALLS.

Inhibition of JAK/STAT signaling sensitizes IL7-dependent samples to dexamethasone

IL7 exerts pro-survival effects by activating JAK1, JAK3 and STAT5 downstream of the IL7R. ${ }^{28}$ We hypothesized that pharmacological inhibition of JAK/STAT signaling would mimic the effects of culturing cells in IL7-deficient media. Ruxolitinib is an FDA approved JAK1/2 inhibitor currently in clinical trials in pediatric patients. ${ }^{29,30}$ We first determined that ruxolitinib blocked IL7induced STAT5 phosphorylation for $48 \mathrm{~h}$ in cultured T-ALL cells (Figures $4 a$ and b). We next exposed T-ALL PDX samples to vehicle, ruxolitinib alone, dexamethasone alone, or dexamethasone plus ruxolitinib. Ruxolitinib alone had only modest effects on cell viability in vitro (Figure 4c). However, combining ruxolitinib with dexamethasone enhanced cell death in the IL7-dependent ETP and non-ETP responder and partial responder samples, but not the nonresponder samples (Figure 4c). Importantly, ruxolitinib increased GC sensitivity in the responder non-ETP T-ALL samples to levels seen in intrinsically GC sensitive T-ALLs (Supplementary Figure S5).

Because JAK3 has also been implicated in IL7R signaling in Tcells, ${ }^{28}$ we tested a newly developed JAK3 inhibitor (JAK3i). ${ }^{17}$ JAK3i inhibited IL7-mediated pSTAT5 induction in a dosedependent manner (Supplementary Figure S6A). Similar to ruxolitinib, JAK3i had only a modest effect as a single agent, but induced GC sensitivity in samples previously shown to use IL7 to resist GCs (Supplementary Figure S6C). These results suggest that both JAK1 and JAK3 are required for functional IL7R signaling and that inhibiting either protein is sufficient to abrogate the protective effect of IL7.

Figure 3. IL7 responsiveness distinguishes non-ETP T-ALL samples and correlates with GC sensitivity. (a) PDX samples were thawed, rested $1 \mathrm{~h}$, and then exposed to vehicle or $100 \mathrm{ng} / \mathrm{ml} \mathrm{IL7}$ for $15 \mathrm{~min}$. STAT5 phosphorylation (pSTAT5) was analyzed by phosphoflow cytometry. Non-ETPs were placed into subsets based upon their IL7 responsiveness. Top depicts representative histograms of PSTAT5 in the basal state (shaded) and after 15 min of stimulation with $100 \mathrm{ng} /$ ml IL7 for each subset (empty line). Bottom shows quantitation of pSTAT5 median fluorescence intensity (MFI) in the basal state and in response to IL7 exposure for each sample. (b) PDX samples were exposed to vehicle or $2.5 \mu \mathrm{m}$ dexamethasone for $48 \mathrm{~h}$ in the presence (black circles) or absence (gray squares) of $25 \mathrm{ng} / \mathrm{ml}$ of IL7. Percentage of viable cells, relative to vehicle, for each of the subgroups (Responders, $n=10$, partial responders, $n=7$, nonresponders, $n=5$, and ETPs, $n=6$ ) was measured by quantification of the Hoechst-negative fraction using flow cytometry. Paired T test (Wilcoxon) was used to evaluate statistical significance. ${ }^{*} P<0.05$, ${ }_{* *} P<0.01$. Data are represented as mean \pm s.e.m. of the biological replicates. Error bars show s.e.m. 
a

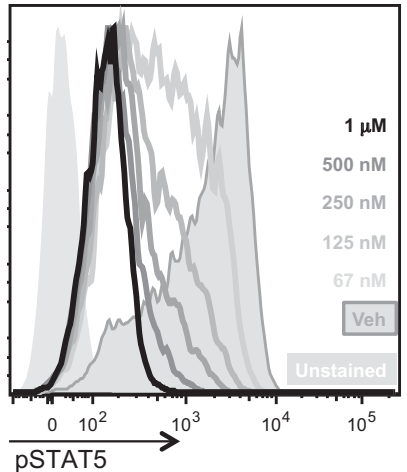

b
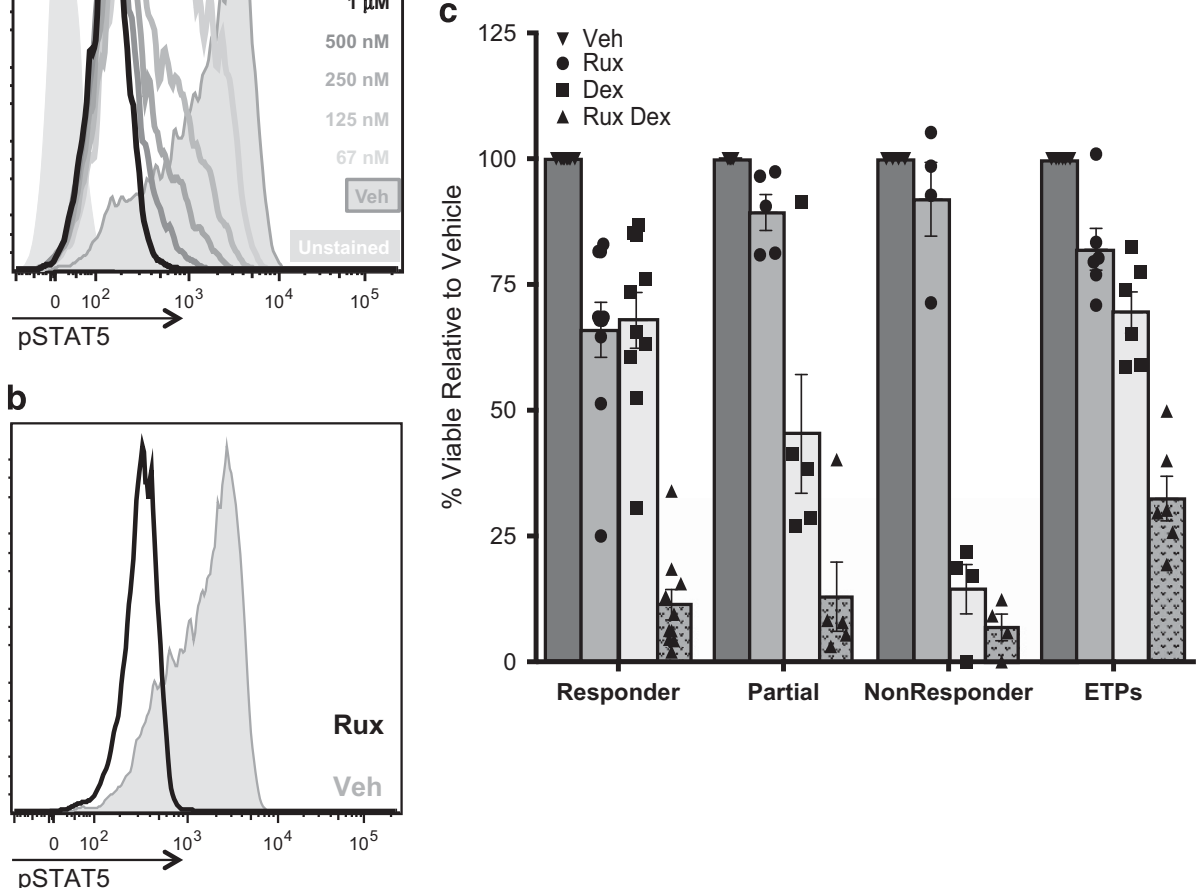

Partial

NonResponder

Figure 4. Ruxolitinib blocks IL7R signaling and abrogates the protective effect of IL7 on GC activity. (a) pSTAT5 of a representative ETP T-ALL PDX sample incubated with increasing doses $(67.5,125,250,500$ and $1000 \mathrm{~nm}$ ) of ruxolitinib (rux) and then stimulated with $100 \mathrm{ng} / \mathrm{ml}$ of IL7 for $15 \mathrm{~min}$. (b) Representative histogram of pSTAT5 after $48 \mathrm{~h}$ of culture with vehicle (tinted line) or $500 \mathrm{~nm}$ ruxolitinib (empty line) in the presence of $25 \mathrm{ng} / \mathrm{ml} \mathrm{IL7}$. (c) PDX samples (Responder $n=10$, Partial $n=5$, non-responder $n=4$, ETP $n=6$ ) were subjected to $48 \mathrm{~h}$ treatment with vehicle, $2.5 \mu \mathrm{m}$ dexamethasone, $500 \mathrm{~nm}$ ruxolitinib or the combination of dexamethasone and ruxolitinib in the presence of $25 \mathrm{ng} / \mathrm{ml}$ IL7. Percentage of viable (Hoechst negative) cells relative to vehicle was quantified for each subset. Data are represented as mean \pm s.e.m. of the biological replicates. Error bars show s.e.m.

The combination of dexamethasone and ruxolitinib alters the balance between BCL2 and BIM

GCs exert their effects by inducing diverse transcriptional changes, which include modulating the expression of many genes involved in apoptosis. In particular, GCs have been described to downregulate the expression of pro-survival $B C L 2$ and upregulate the expression of pro-apoptotic BIM in lymphoblasts. ${ }^{9,10,31}$ IL7R signaling affects expression of anti-apoptotic genes which induces expression of pro-survival proteins including $B C L 2$, among others. $^{32-34}$ To study potential molecular events that might mediate IL7-dependent GC resistance, we compared BCL2 and BIM protein expression between IL7-dependent GC-resistant ETP and non-ETP leukemias, and IL7-independent GC-resistant ETP samples exposed to vehicle, ruxolitinib alone, dexamethasone alone, or dexamethasone plus ruxolitinib in the presence of IL7. Gating on only viable (caspase- 3 negative) cells (Supplementary Figure S7A and B) after $48 \mathrm{~h}$ of drug exposure, we found that IL7independent GC-resistant ETPs had minimal changes in BCL2 or BIM expression in any condition. In contrast, in IL7-dependent GCresistant leukemias, ruxolitinib treatment alone resulted in a marked decrease in BCL2 protein expression relative to vehicle, while cells surviving dexamethasone treatment alone had elevated BCL2 expression. Addition of ruxolitinib to ruxolitinib alone, dexamethasone abrogated the increase in BCL2 (Figure 5a). Given this unexpected increase in $\mathrm{BCL}-2$ expression induced by dexamethasone treatment in the presence of IL7, we assessed $\mathrm{BCL}-2$ transcript levels under these conditions, further confirming this finding (Supplementary Figure 7A). Interestingly, dexamethasone resulted in a significant increase in BIM expression, while ruxolitinib had no effect alone or in combination with dexamethasone (Figure 5b).

To interrogate the functional consequences of these changes in protein expression, we utilized dynamic $\mathrm{BH} 3$ profiling to assess the intrinsic apoptotic potential, or 'priming', of cells under these different conditions. We first measured priming using a synthetic peptide corresponding to the BIM BH3 domain, which antagonizes all anti-apoptotic members of the BCL2 family of proteins. ${ }^{35}$ As a single agent, ruxolitinib, but not dexamethasone, increased apoptotic priming in IL7-dependent GC-resistant samples. Combination therapy further augmented apoptotic priming in these leukemias, supporting their IL7-dependent, GC-resistant status. (Figure 6a). Consistent with their IL7-independent GC-resistant status, apoptotic priming was not affected in any treatment conditions in these samples. To determine if differences in apoptotic potential were attributable specifically to alterations in BCL2 activity, consistent with the changes in protein expression observed in Figure 5, we performed dynamic $\mathrm{BH} 3$ profiling using the BCL2 antagonist ABT-199. Importantly, the profile appeared highly similar to that of the BIM BH3 peptide, suggesting that IL7associated differences in apoptotic potential are driven in large part by a BCL2-dependent mechanism (Figure 6b).

Finally, we asked whether ABT-199 could mimic the ability of ruxolitinib to induce GC sensitivity in IL7-dependent T-ALLs. Cells were treated in the presence of IL7 with dexamethasone alone or in combination with either ruxolitinib or ABT-199. Consistent with our previous findings, both IL7-dependent and -independent samples were dexamethasone resistant in the presence of IL7. Whereas addition of ruxolitinib markedly and selectively 
a

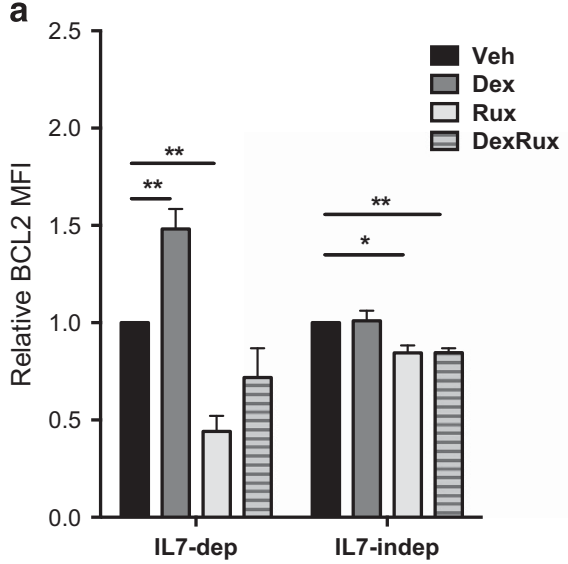

b

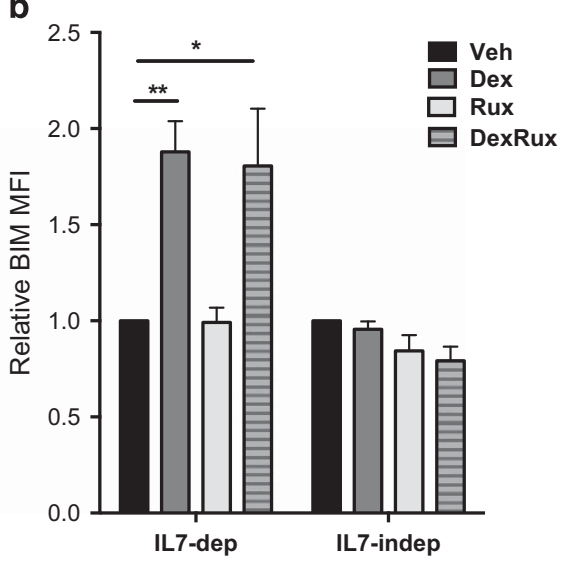

Figure 5. Treatment with ruxolitinib and dexamethasone alters the balance between BCL2 and BIM. Representative PDX samples that either need IL7 to survive GC-induced death ('IL7-dependent', $n=5$ ) or that were GC-resistant (remained $>75 \%$ viable) even in the absence of IL7 ('IL7-independent', $n=4$ ), were treated for $48 \mathrm{~h}$ with vehicle, $2.5 \mu \mathrm{m}$ dexamethasone, $500 \mathrm{~nm}$ ruxolitinib or the combination of both drugs in IL7-containing media. Expression of BCL2 (a) and BIM (b) was determined for cells in the viable, active caspase- 3 negative gate. One sample $t$ test was used to evaluate statistical significance. ${ }^{*} P<0.05 ;{ }^{*} P<0.01$. Data are represented as mean \pm s.e.m. of the biological replicates. Error bars show s.e.m.

attenuated dexamethasone resistance in IL7-dependent GCresistant leukemias, ABT-199 had more modest effects (Figure 6c). These data suggest that IL7-dependent dexamethasone resistance is partially due to changes in BCL2 activity.

To test the hypothesis that PI3K/AKT signaling might link IL7R activation to dexamethasone resistance, we exposed IL7dependent GC-resistant leukemias to the AKT inhibitor MK2206, alone and in combination with ruxolitinib and/or dexamethasone, but observed little or no effect on T-ALL survival and apoptotic priming (Supplementary Figure S8B and D). Although AKT inhibition resulted in the downregulation of $B C L 2$, in contrast to the net effect of dexamethasone plus ruxolitinib on $B C L 2$ expression, the combination of MK2206 with dexamethasone was not sufficient to counteract GC-induced upregulation of BCL2.

\section{DISCUSSION}

The goal of modern pediatric ALL therapy is to provide sufficient therapy to maximize cure while minimizing toxicity. Given that most T-ALL relapses occur early and that salvage rates are dismal following relapse, ${ }^{3}$ characterizing mechanisms of intrinsic or acquired chemotherapy resistance are essential for improving outcomes. Currently, the strongest predictors of outcome in T-ALL are MRD and the initial response to GCs during induction therapy. 5,6 Previous reports demonstrate that in vitro drug sensitivity correlates with in vivo clinical response. ${ }^{20-22}$ While further studies are needed to define biomarkers of clinical response and resistance to GC therapy, our data suggest that, at least in vitro, differences in GC sensitivity are independent of GR expression. Furthermore, we have identified a subset of diagnostic T-ALL samples that is GC resistant in an IL7-dependent manner and that can be sensitized to GCs with JAK inhibition. These findings have important translational implications for considering the addition of IL7R pathway inhibitors in a subset of patients.

Here we show that PDX samples derived from patients with ETP T-ALL are more resistant to in vitro dexamethasone treatment than are non-ETP T-ALL PDX samples. This is consistent with the observation that ETP T-ALL patients frequently have positive MRD at the end of induction, ${ }^{36-38}$ likely reflecting, at least in part, intrinsic GC resistance. Similarly, non-malignant double negative (DN) thymocytes, which give rise to ETP T-ALLs, are refractory to GCs. ${ }^{39}$ This appears to be due to IL7-mediated upregulation of the pro-survival factor $\mathrm{BCL} 2{ }^{32}$ which is more highly expressed in DN cells relative to other thymocyte populations. ${ }^{40,41}$ We have identified highly consistent patterns of GC resistance, IL7 responsiveness, and high $\mathrm{BCL} 2$ expression across ETP T-ALL samples, ${ }^{16}$ supporting the hypothesis that these leukemias retain properties of the DN thymocytes from which they arise.

The genetic heterogeneity of T-ALL has precluded the identification of clinically relevant genetic subsets that are amenable to therapeutic intervention. Despite this heterogeneity, we reasoned that differences in sensitivity to chemotherapy might result from a more limited set of changes in signal transduction and response to environmental stimuli. Supporting this notion, ETP T-ALLs were uniformly GC resistant while non-ETP T-ALLs were much more heterogeneous. We could identify distinct subsets within these non-ETP samples based upon their response to IL7 stimulation. Importantly, mutational analysis revealed that IL7dependent GC resistance is independent of the presence of activating pathway mutations, suggesting that biochemical analysis may be necessary for the identification of distinct T-ALL subsets that may carry prognostic and therapeutic significance.

We speculate that a subset of T-ALLs may retain physiologic dependence on IL7. IL7 is a well-established pro-survival factor present in plasma and lymphopoietic niches ${ }^{42}$ that protects normal thymocytes and T-ALL cells from spontaneous ${ }^{18,43,44}$ and chemotherapy-induced apoptosis. ${ }^{15,45}$ Since lymphocyte depletion results in increased IL7 production by stromal cells as a means of restoring lymphocyte homeostasis, ${ }^{46-49}$ we hypothesize that there may exist a positive feedback loop, whereby the majority of leukemia cells are depleted in response to GCs, but those cells that survive the initial exposure to GCs then encounter augmented levels of IL7 in the microenvironment, which, in the case of patients with IL7-induced GC resistance, could facilitate the persistence of these cells. While prior work suggested that elevated IL7R expression positively correlates with clinical outcomes, ${ }^{18,50}$ we and others ${ }^{15,45,51}$ have found a negative correlation between IL7R signaling and GC sensitivity. We expand on this finding here by demonstrating a novel therapeutic strategy to ameliorate this antagonistic relationship between IL7R signaling and GC sensitivity.

We hypothesized that IL7-dependent dexamethasone resistance could be overcome with inhibition of IL7R signaling. All signaling initiated by IL7 requires phosphorylation of the IL7R by constitutively associated JAK kinases, which in turn creates docking sites that allow for the recruitment of effectors such as STAT5 and PI3K.52 JAK inhibition would therefore prevent IL7induced activation of both pro-survival pathways. The JAK1/2 

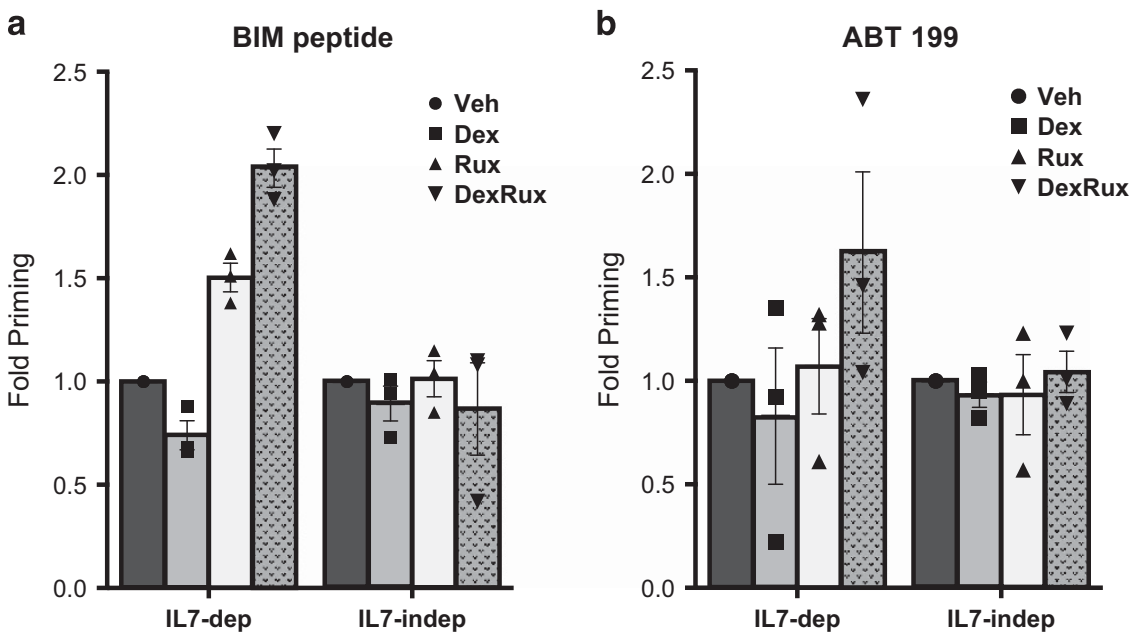

C

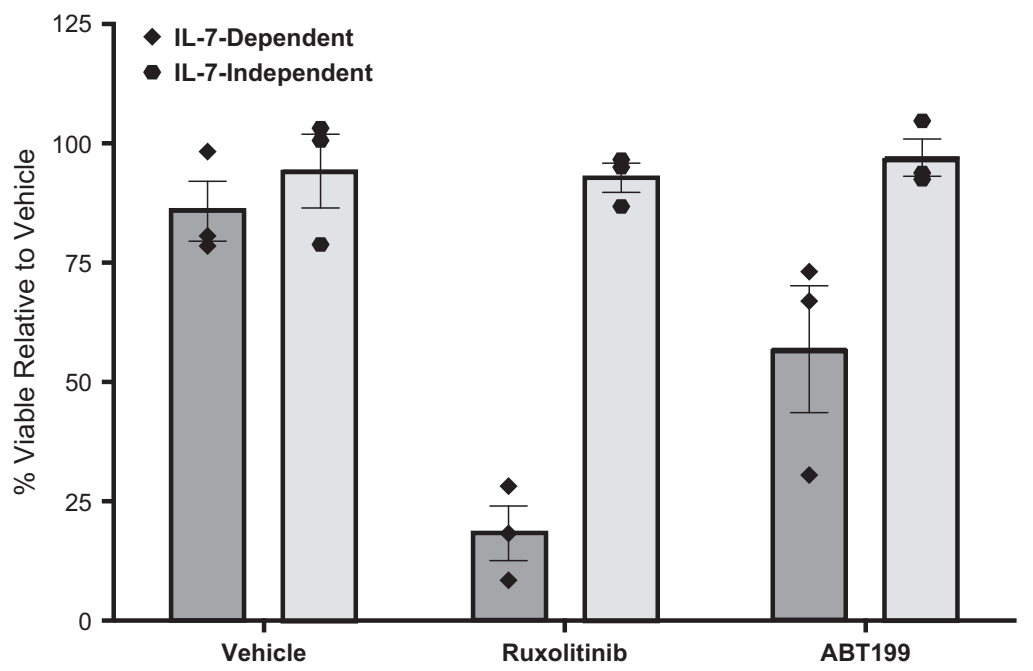

Figure 6. IL7R signaling prevents a dexamethasone-induced increase in apoptotic priming. (a) Apoptotic priming of IL7-dependent ( $n=3$ ) and -independent $(n=3)$ samples treated for $16 \mathrm{~h}$ with vehicle, $1 \mu \mathrm{m}$ dexamethasone, $500 \mathrm{~nm}$ ruxolitinib, or a combination of the two in IL7containing media, as assessed by depletion of intracellular cytochrome c following a 1-h exposure to $100 \mathrm{nM}$ BIM BH3 peptide. \% priming values are normalized to the vehicle control condition. (b) Apoptotic priming of IL7-dependent and -independent samples following a 1-h exposure to $1 \mu \mathrm{M}$ ABT-199. \% priming values are normalized to the vehicle control condition. (c) Cell viability of IL7-dependent and -independent samples with the addition of $2.5 \mu \mathrm{m}$ dexamethasone relative to vehicle, $500 \mathrm{~nm}$ ruxolitinib, or $200 \mathrm{~nm}$ ABT-199 alone. Data are represented as mean \pm s.e.m. of the biological replicates. Error bars show s.e.m.

inhibitor ruxolitinib, an FDA approved agent that is well tolerated in pediatric patients, ${ }^{30}$ prevents IL7R-dependent phosphorylation of both AKT ${ }^{53,54}$ and STAT5. ${ }^{16}$ Pre-clinical studies of ruxolitinib using in vivo models of mouse ${ }^{55}$ and human ${ }^{16}$ ETP-ALL demonstrated an anti-leukemic effect. Importantly, as a single agent, ruxolitinib did not induce complete remissions, suggesting a cytostatic, rather than cytotoxic, effect. However, combination with standard chemotherapy may potentiate the anti-leukemic effect of these conventional therapies, as seen in Ph+ ALL. ${ }^{56}$ We similarly observe in this panel of T-ALLs that, while ruxolitinib alone is not cytotoxic in vitro in most samples, it is capable of sensitizing cells to dexamethasone-induced apoptosis. A similar effect was observed with JAK3i, providing evidence that both JAK1 and JAK3 are required for effective IL7R signaling. Interestingly, of the agents we tested, the protective effect of IL7 was restricted to dexamethasone and vorinostat, agents that modulate gene transcription. To our surprise, JAK inhibition did not overcome GC resistance in some ETP T-ALLs, despite their strong response to IL7 stimulation, suggesting that they utilize IL7independent means to resist GCs. Alternative therapeutic strategies to improve GC efficacy for this subset of patients would be needed.

In normal thymocytes, IL7 mediates survival by inducing expression of $\mathrm{BCL} 2,{ }^{32}$ among other pro-survival proteins. ${ }^{33,34}$ $\mathrm{GCs}$ are known to downregulate expression of $\mathrm{BCL} 2,{ }^{31,57}$ but here we show that, in the IL7-dependent GC-resistant samples, the cells that survive dexamethasone treatment show elevated levels of BCL2. We hypothesize that this is an effect of IL7R signaling, and demonstrate, using both protein expression and a functional assay, that the effects of IL7R and GCs on BCL2 can be uncoupled via inhibition of IL7R signaling. Specifically, we demonstrate that, in IL7-dependent leukemias, the IL7-induced upregulation of BCL2 overcomes the GC-induced downregulation of $\mathrm{BCL} 2$, resulting in a persistent anti-apoptotic state. We demonstrate the functional significance of this IL7 effect by showing that dexamethasone increases the apoptotic potential of these cells, and specifically decreases BCL2 activity, only in the absence of intact IL7R signaling. The fact that the combination of dexamethasone with ABT-199 failed to completely sensitize IL7-dependent samples to GC-induced apoptosis might be explained by the activity of other pro-survival proteins, such as MCL1 or $\mathrm{BCL}-\mathrm{XL},{ }^{33,34}$ which have 
similarly been shown to contribute to GC resistance. Additional studies are needed to evaluate the role of these other key antiapoptotic mediators in GC resistance in T-ALL.

In conclusion, we identify biologically distinct groups of pediatric T-ALLs that differ with respect to intrinsic GC sensitivity in the presence of IL7 and demonstrate that this correlates with STAT5 activation in response to IL7 stimulation. A prospective analysis to confirm the existence of these subsets is being performed in the context of the current COG frontline trial AALL1231. Our data suggest that individual T-ALL samples have intrinsic differences in GC sensitivity, and show that this sensitivity can be altered by environmental stimuli, such as IL7. Modulating sensitivity to GCs with agents such as ruxolitinib or JAK3i in the subset of patients with IL7-dependent GC resistance should be explored as a means of facilitating a deeper, more durable remission and as a way of ultimately improving outcomes.

\section{CONFLICT OF INTEREST}

The authors declare no conflict of interest.

\section{ACKNOWLEDGEMENTS}

This work was supported by National Cancer Institute Grant R01 11666503 (DT, MLH TMH and BLW), the Pepp Family Foundation (MLH), St Baldrick's Foundation, and Campini Foundation. The authors are indebted to all patients and their physicians who participated in the study. We thank Mignon Loh and Kevin Shannon for critical reading of the manuscript, and Ernesto Diaz-Flores for helpful discussion and advice.

\section{AUTHOR CONTRIBUTIONS}

CDM and LKM designed the study, analyzed the data and wrote the manuscript. $\mathrm{BJH}, \mathrm{KAS}, \mathrm{MSZ}$ and JVN contributed to study design and data analysis. BJH, KAS, MSZ, JVN, GAS, JT, JRR, MAK and BLW contributed to data acquisition and manuscript editing. TMH, DTT and BLW contributed to study design and manuscript editing. MLH designed and interpreted the experimental work and wrote the manuscript.

\section{REFERENCES}

1 Graux C, Cools J, Michaux L, Vandenberghe P, Hagemeijer A. Cytogenetics and molecular genetics of T-cell acute lymphoblastic leukemia: from thymocyte to lymphoblast. Leukemia 2006; 20: 1496-1510.

2 Hunger SP, Lu X, Devidas M, Camitta BM, Gaynon PS, Winick NJ et al. Improved survival for children and adolescents with acute lymphoblastic leukemia between 1990 and 2005: a report from the children's oncology group. J Clin Oncol 2012; 30: 1663-1669.

3 Bhojwani D, Pui C-H. Relapsed childhood acute lymphoblastic leukaemia. Lancet Oncol 2013; 14: e205-e217.

4 Coustan-Smith E, Mullighan CG, Onciu M, Behm FG, Raimondi SC, Pei D et al. Early T-cell precursor leukaemia: a subtype of very high-risk acute lymphoblastic leukaemia. Lancet Oncol 2009; 10: 147-156.

5 Dördelmann M, Reiter A, Borkhardt A, Ludwig WD, Götz N, Viehmann S et al. Prednisone response is the strongest predictor of treatment outcome in infant acute lymphoblastic leukemia. Blood 1999; 94: 1209-1217.

6 Riehm H, Reiter A, Schrappe M, Berthold F, Dopfer R, Gerein V et al. [Corticosteroid-dependent reduction of leukocyte count in blood as a prognostic factor in acute lymphoblastic leukemia in childhood (therapy study ALL-BFM 83)]. Klin Padiatr 1987; 199: 151-160.

7 Kaspers GJL, Wijnands JJM, Hartmann R, Huismans L, Loonen AH, Stackelberg A et al. Immunophenotypic cell lineage and in vitro cellular drug resistance in childhood relapsed acute lymphoblastic leukaemia. Eur J Cancer 2005; 41: 1300-1303.

8 Klumper E, Pieters R, Veerman AJ, Huismans DR, Loonen AH, Hählen $\mathrm{K}$ et al. In vitro cellular drug resistance in children with relapsed/refractory acute lymphoblastic leukemia. Blood 1995; 86: 3861-3868.

9 Yamamoto KR. Steroid receptor regulated transcription of specific genes and gene networks. Annu Rev Genet 1985; 19: 209-252.

10 Schmidt S, Rainer J, Ploner C, Presul E, Riml S, Kofler R. Glucocorticoid-induced apoptosis and glucocorticoid resistance: molecular mechanisms and clinical relevance. Cell Death Differ 2004; 11(Suppl 1): S45-555.
11 Tissing WJE, Meijerink JPP, den Boer ML, Brinkhof B, van Rossum EFC, van Wering ER et al. Genetic variations in the glucocorticoid receptor gene are not related to glucocorticoid resistance in childhood acute lymphoblastic leukemia. Clin Cancer Res 2005; 11: 6050-6056.

12 Irving JAE, Minto L, Bailey S, Hall AG. Loss of heterozygosity and somatic mutations of the glucocorticoid receptor gene are rarely found at relapse in pediatric acute lymphoblastic leukemia but may occur in a subpopulation early in the disease course. Cancer Res 2005; 65: 9712-9718.

13 Piovan E, Yu J, Tosello V, Herranz D, Ambesi-Impiombato A, Da Silva AC et al. Direct reversal of glucocorticoid resistance by AKT inhibition in acute lymphoblastic leukemia. Cancer Cell 2013; 24: 766-776.

14 Marke R, Havinga J, Cloos J, Demkes M, Poelmans G, Yuniati L et al. Tumor suppressor IKZF1 mediates glucocorticoid resistance in B-cell precursor acute lymphoblastic leukemia. Leukemia 2016; 30: 1599-1603.

15 Wuchter C, Ruppert V, Schrappe M, Dörken B, Ludwig W-D, Karawajew L. In vitro susceptibility to dexamethasone- and doxorubicin-induced apoptotic cell death in context of maturation stage, responsiveness to interleukin 7, and early cytoreduction in vivo in childhood T-cell acute lymphoblastic leukemia. Blood 2002; 99: 4109-4115.

16 Maude SL, Dolai S, Delgado-Martin C, Vincent T, Robbins A, Selvanathan A et al. Efficacy of JAK/STAT pathway inhibition in murine xenograft models of early T-cell precursor (ETP) acute lymphoblastic leukemia. Blood 2015; 125: 1759-1767.

17 Smith GA, Uchida K, Weiss A, Taunton J. Essential biphasic role for JAK3 catalytic activity in IL-2 receptor signaling. Nat Chem Biol 2016; 12: 373-379.

18 Karawajew L, Ruppert V, Wuchter C, Kösser A, Schrappe M, Dörken B et al. Inhibition of in vitro spontaneous apoptosis by IL-7 correlates with bcl-2 up-regulation, cortical/mature immunophenotype, and better early cytoreduction of childhood T-cell acute lymphoblastic leukemia. Blood 2000; 96: 297-306.

19 Ryan J, Letai A. BH3 profiling in whole cells by fluorimeter or FACS. Methods 2013; 61: 156-164.

20 Pieters R, Huismans DR, Loonen AH, Hählen K, van der Does-van den Berg A, van Wering ER et al. Relation of cellular drug resistance to long-term clinical outcome in childhood acute lymphoblastic leukaemia. Lancet 1991; 338: 399-403.

21 Kaspers GJ, Veerman AJ, Pieters R, Van Zantwijk CH, Smets LA, Van Wering ER et al. In vitro cellular drug resistance and prognosis in newly diagnosed childhood acute lymphoblastic leukemia. Blood 1997; 90: 2723-2729.

22 Den Boer ML, Harms DO, Pieters R, Kazemier KM, Gobel U, Körholz D et al. Patient stratification based on prednisolone-vincristine-asparaginase resistance profiles in children with acute lymphoblastic leukemia. J Clin Oncol 2003; 21: 3262-3268.

23 Schmitz M, Breithaupt P, Scheidegger N, Cario G, Bonapace L, Meissner B et al. Xenografts of highly resistant leukemia recapitulate the clonal composition of the leukemogenic compartment. Blood 2011; 118: 1854-1864.

24 Liem NLM, Papa RA, Milross CG, Schmid MA, Tajbakhsh M, Choi S et al. Characterization of childhood acute lymphoblastic leukemia xenograft models for the preclinical evaluation of new therapies. Blood 2004; 103: 3905-3914.

25 Jiang Q, Li WQ, Aiello FB, Mazzucchelli R, Asefa B, Khaled AR et al. Cell biology of IL-7, a key lymphotrophin. Cytokine Growth Factor Rev 2005; 16: 513-533.

26 Silva A, Laranjeira ABA, Martins LR, Cardoso BA, Demengeot J, Yunes JA et al. IL-7 contributes to the progression of human T-cell acute lymphoblastic leukemias. Cancer Res 2011; 71: 4780-4789.

27 Winter SS, Dunsmore KP, Devidas M, Eisenberg N, Asselin BL, Wood BL et al. Safe integration of nelarabine into intensive chemotherapy in newly diagnosed T-cell acute lymphoblastic leukemia: Children's Oncology Group Study AALL0434. Pediatr Blood Cancer 2015; 62: 1176-1183.

28 Foxwell BM, Beadling C, Guschin D, Kerr I, Cantrell D. Interleukin-7 can induce the activation of Jak 1, Jak 3 and STAT 5 proteins in murine T cells. Eur J Immunol 1995; 25: 3041-3046.

29 Quintás-Cardama A, Vaddi K, Liu P, Manshouri T, Li J, Scherle PA et al. Preclinical characterization of the selective JAK1/2 inhibitor INCB018424: therapeutic implications for the treatment of myeloproliferative neoplasms. Blood 2010; 115: 3109-3117.

30 Loh ML, Tasian SK, Rabin KR, Brown P, Magoon D, Reid JM et al. A phase 1 dosing study of ruxolitinib in children with relapsed or refractory solid tumors, leukemias, or myeloproliferative neoplasms: A Children's Oncology Group phase 1 consortium study (ADVL1011). Pediatr Blood Cancer 2015; 62: 1717-1724.

31 Jing D, Bhadri VA, Beck D, Thoms JAl, Yakob NA, Wong JWH et al. Opposing regulation of $\mathrm{BIM}$ and $\mathrm{BCL} 2$ controls glucocorticoid-induced apoptosis of pediatric acute lymphoblastic leukemia cells. Blood 2015; 125: 273-283.

32 Akashi K, Kondo M, von Freeden-Jeffry U, Murray R, Weissman IL. Bcl-2 rescues T lymphopoiesis in interleukin-7 receptor-deficient mice. Cell 1997; 89: 1033-1041.

33 Amos CL, Woetmann A, Nielsen M, Geisler C, Odum N, Brown BL et al. The role of caspase 3 and $B c l x L$ in the action of interleukin 7 (IL-7): a survival factor in activated human T cells. Cytokine 1998; 10: 662-668. 
34 Opferman JT, Letai A, Beard C, Sorcinelli MD, Ong CC, Korsmeyer SJ. Development and maintenance of $B$ and $T$ lymphocytes requires antiapoptotic MCL-1. Nature 2003; 426: 671-676.

35 Letai A, Bassik MC, Walensky LD, Sorcinelli MD, Weiler S, Korsmeyer SJ. Distinct $\mathrm{BH} 3$ domains either sensitize or activate mitochondrial apoptosis, serving as prototype cancer therapeutics. Cancer Cell. 2002; 2: 183-192.

36 Wood BL, Winter SS, Dunsmore KP, Devidas M, chen si, Asselin BL et al. T-lymphoblastic leukemia (T-ALL) shows esscellent outcome, lack of significance of the early thymic precursor (ETP) immunophenotype, and validation of the prognostic value of end-induction minimal residual disease (MRD) in Children's Oncology Group (COG) study AALL0434. Blood 2014; 124: 1-1. (Abstract 1).

37 Patrick K, Wade R, Goulden N, Mitchell C, Moorman AV, Rowntree C et al. Outcome for children and young people with Early T-cell precursor acute lymphoblastic leukaemia treated on a contemporary protocol, UKALL 2003. Br J Haematol 2014; 166: 421-424.

38 Conter V, Valsecchi MG, Buldini B, Parasole R, Locatelli F, Colombini A et al. Early T-cell precursor acute lymphoblastic leukaemia in children treated in AIEOP centres with AIEOP-BFM protocols: a retrospective analysis. Lancet Haematol 2016; 3: e80-e86.

39 Berki T, Pálinkás L, Boldizsár F, Németh P. Glucocorticoid (GC) sensitivity and GC receptor expression differ in thymocyte subpopulations. Int Immunol 2002; 14: 463-469.

40 Veis DJ, Sentman CL, Bach EA, Korsmeyer SJ. Expression of the Bcl-2 protein in murine and human thymocytes and in peripheral T lymphocytes. J Immunol 1993; 151: 2546-2554.

41 Gratiot-Deans J, Ding L, Turka LA, Nuñez G. bcl-2 proto-oncogene expression during human $T$ cell development. Evidence for biphasic regulation. J Immunol 1993; 151: 83-91.

42 Kim GY, Hong C, Park J-H. Seeing is believing: illuminating the source of in vivo interleukin-7. Immune Netw 2011; 11: 1-10.

43 Barata JT, Cardoso AA, Nadler LM, Boussiotis VA. Interleukin-7 promotes survival and cell cycle progression of T-cell acute lymphoblastic leukemia cells by down-regulating the cyclin-dependent kinase inhibitor p27(kip1). Blood 2001; 98: 1524-1531.

44 Barata JT, Cardoso AA, Boussiotis VA. Interleukin-7 in T-cell acute lymphoblastic leukemia: an extrinsic factor supporting leukemogenesis? Leuk Lymphoma 2005; 46: 483-495.

45 Hernández-Caselles T, Martínez-Esparza M, Sancho D, Rubio G, Aparicio $P$. Interleukin-7 rescues human activated T lymphocytes from apoptosis induced by glucocorticoesteroids and regulates bcl-2 and CD25 expression. Hum Immunol 1995; 43: 181-189.

46 Bolotin E, Annett G, Parkman R, Weinberg K. Serum levels of IL-7 in bone marrow transplant recipients: relationship to clinical characteristics and lymphocyte count. Bone Marrow Transplant. 1999; 23: 783-788.

47 Napolitano LA, Grant RM, Deeks SG, Schmidt D, De Rosa SC, Herzenberg LA et al. Increased production of IL-7 accompanies HIV-1-mediated T-cell depletion: implications for T-cell homeostasis. Nat Med 2001; 7: 73-79.
48 Zubkova I, Mostowski H, Zaitseva M. Up-regulation of IL-7, stromal-derived factor1 alpha, thymus-expressed chemokine, and secondary lymphoid tissue chemokine gene expression in the stromal cells in response to thymocyte depletion: implication for thymus reconstitution. J Immunol 2005; 175: 2321-2330.

49 Miller CN, Hartigan-O'Connor DJ, Lee MS, Laidlaw G, Cornelissen IP, Matloubian M et al. IL-7 production in murine lymphatic endothelial cells and induction in the setting of peripheral lymphopenia. Int Immunol 2013; 25: 471-483.

50 Cleaver AL, Beesley AH, Firth MJ, Sturges NC, O'Leary RA, Hunger SP et al. Genebased outcome prediction in multiple cohorts of pediatric T-cell acute lymphoblastic leukemia: a Children's Oncology Group study. Mol Cancer 2010; 9: 105.

51 Li Y, Buijs-Gladdines JGCAM, Canté-Barrett K, Stubbs AP, Vroegindeweij EM, Smits WK et al. IL-7 receptor mutations and steroid resistance in pediatric $t$ cell acute lymphoblastic leukemia: A Genome Sequencing Study. PLoS Med 2016; 13: e1002200.

52 Hofmeister R, Khaled AR, Benbernou N, Rajnavolgyi E, Muegge K, Durum SK. Interleukin-7: physiological roles and mechanisms of action. Cytokine Growth Factor Rev 1999; 10: 41-60.

53 Zenatti PP, Ribeiro D, Li W, Zuurbier L, Silva MC, Paganin M et al. Oncogenic IL7R gain-of-function mutations in childhood T-cell acute lymphoblastic leukemia. Nat Genet 2011; 43: 932-939.

54 Canté-Barrett K, Spijkers-Hagelstein JaP, Buijs-Gladdines JGC a. M, Uitdehaag JCM, Smits WK, van der Zwet J et al. MEK and PI3K-AKT inhibitors synergistically block activated IL7 receptor signaling in T-cell acute lymphoblastic leukemia. Leukemia 2016; 30: 1832-1843.

55 Treanor LM, Zhou S, Janke L, Churchman ML, Ma Z, Lu T et al. Interleukin-7 receptor mutants initiate early $\mathrm{T}$ cell precursor leukemia in murine thymocyte progenitors with multipotent potential. J Exp Med 2014; 211: 701-713.

56 Schultz KR, Bowman WP, Aledo A, Slayton WB, Sather H, Devidas M et al. Improved early event-free survival with imatinib in Philadelphia chromosomepositive acute lymphoblastic leukemia: a children's oncology group study. J Clin Oncol 2009; 27: 5175-5181.

57 Rogatsky I, Hittelman AB, Pearce D, Garabedian MJ. Distinct glucocorticoid receptor transcriptional regulatory surfaces mediate the cytotoxic and cytostatic effects of glucocorticoids. Mol Cell Biol 1999; 19: 5036-5049.

(2) $⿴$ This work is licensed under a Creative Commons AttributionBY NC ND NonCommercial-NoDerivs 4.0 International License. The images or other third party material in this article are included in the article's Creative Commons license, unless indicated otherwise in the credit line; if the material is not included under the Creative Commons license, users will need to obtain permission from the license holder to reproduce the material. To view a copy of this license, visit http:// creativecommons.org/licenses/by-nc-nd/4.0/

(c) The Author(s) 2017

Supplementary Information accompanies this paper on the Leukemia website (http://www.nature.com/leu) 